\title{
Using a task analysis approach within a guided problem-solving model to design mathematical learning activities
}

\author{
Aneshkumar Maharaj \\ School of Mathematical Sciences, University of KwaZulu-Natal \\ Email: maharaja32@ukzn.ac.za
}

The FET Curriculum Statements for Mathematics advocates that knowledge integrates theory, skills and values. This paper focuses on a guided problem-solving teaching model that provides a framework to do this. A task analysis approach is used within this framework to illustrate how educators could frame mathematical questions based on the relevant mathematical content.

\section{Introduction}

An analysis of matriculation examiners' reports (KwaZulu-Natal Department of Education, 2000, 2001 \& 2002; Gauteng Department of Education, 2000, 2001, $2002 \&$ 2003) indicated a number of recurring issues evident in candidates' work that lead to poor performance:

- a poor understanding of mathematical terminology and concepts;

- an inability to recall and apply formulae, and poor substitution skills;

- an inability to recall and apply algorithms;

- a lack of manipulative skills which relate to simplifying arithmetic, algebraic and trigonometric expressions, and solving of polynomial equations of degree two and three;

- an inability to answer interpretative questions;

- a poor knowledge of book-work, in particular statements of and proofs of theorems or rules in the context of given diagrams.

These were reflected by the candidates' responses to the sorts of questions that are typical of those that appear in examination papers (Box 1). To solve the equation in the first question a learner should be able to identify it as a quadratic equation and then recall and apply a suitable algorithm, for example, the factorisation technique for solving a quadratic equation. The second question requires an interpretation of the question together with required manipulative skills to transform the given equation to the equivalent centre-radius form, an integration of relevant knowledge from algebra and geometry supported by appropriate visualisation. These manipulative skills are required to deduce the embedded information from what is given. For the third question, a requirement is that the given information (in symbolic form) and figure be used to prove the theorem which states that if two triangles are equiangular then the corresponding sides are in proportion.
This paper is based on a number of assumptions regarding the learning of mathematics:

- Learning mathematics requires the mastery of concepts, relationships - which include conventions such as $\sqrt{3}$ - and algorithms, and their application.

- Learning proceeds from the known to the unknown.

- There are networks of concepts in the brain which aid understanding, so learning should result in a gradual expansion and modification of these networks. The latter is premised on the work of Hiebert and Carpenter (1992) who presented a theory of understanding based on the formation and interplay of internal and external representations.

- Learning of a mathematical topic involves "many actions, processes, and objects that need to be organised and linked into a coherent framework, which is called a schema" (Dubinsky, Weller, McDonald \& Brown, 2005). The authors refer to this as the APOS (actions, processes, objects and schema) approach.

- It is possible to plan for meaningful teaching of mathematics by simultaneously addressing the cognitive and affective domains (Cangelosi, 1996), by focusing on the actions, processes, objects and schema (APOS).

- An APOS analysis of a topic could give insight into the type of thinking that an individual might be capable of. It does not indicate what happens in an individual's mind since this is unknowable.

This paper focuses on addressing the following question, beginning with the assumptions above: how might the educator work on tasks so as to promote mathematical learning and address the recurring problematic issues indicated in the matriculation examiners' reports? 
1. Solve for $x: 3 x^{2}+2 x=5$

2. Determine the equation of the tangent to the circle $x^{2}-2 x+y^{2}+4 y=5$ at the point $(-2 ;-1)$.

3. Refer to the figure.
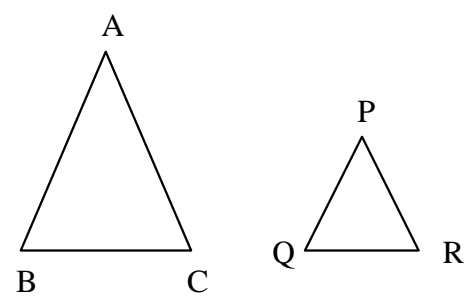

In triangles $A B C$ and $P Q R$,

$\hat{A}=\hat{P}, \hat{B}=\hat{Q}$ and $\hat{C}=\hat{R}$.

Copy the sketch in your answer book and use it to prove the theorem that states

$\frac{P Q}{A B}=\frac{P R}{A C}=\frac{Q R}{B C}$.

Box 1. Typical examination questions.

In trying to find an answer to this question, this paper will address three aspects. Firstly, it discusses an interpretation of Cangelosi's problemsolving model. Secondly, it illustrates how this model can be used to promote teaching and learning of mathematics, and finally, it shows how a task analysis approach can be used within this model to formulate mathematical activities. These activities are aimed at developing competencies required of mathematics educators, as implied in the FET Curriculum Statements for Mathematics (Department of Education, 2003).

The structure of these activities is based on work done by Mason (2000). As such, the framing of questions and activities is guided by the following conjecture: If learners are both led through and invited to participate in typical mathematical activities, then they are more likely to appreciate mathematics as a discipline. This helps to focus on both the cognitive and affective domains as noted in the assumptions. It is particularly helpful for designing activities which elicit in the learner a willingness to try and appreciation of the relevant mathematical concept, during the different reasoning phases of the guided problem-solving teaching model (Figure 1).

\section{The guided problem-solving model}

The guided problem-solving model, which is an interpretation of Cangelosi's (1996) problemsolving model, is illustrated in Figure 1. This approach has three phases:

1) Inductive reasoning (conceptual phase);

2) Inductive and deductive reasoning (simple knowledge and knowledge of a process phase);

3) Deductive reasoning (application phase).

It is important to note that there is always an interplay between inductive and deductive reasoning. They occur continuously and are in a constant iterative relationship in mathematical thinking. For example, in an inductive process, there is frequently a preliminary 'generalising' step. A conclusion or the finalising of the inductive aspect forms the beginning of the deductive aspect. Generalising at each of the different phases implies that a deductive mode of reasoning comes into play. Furthermore, applications or the solving of problems requires knowledge of algorithms, and could lead to the discovery of new relationships. Hence, there is an interplay between phases (see Figure 1).

In the inductive reasoning phase, inductive learning activities should be used to construct a concept or discover a discoverable relationship. Instruction through guided inquiry could help 


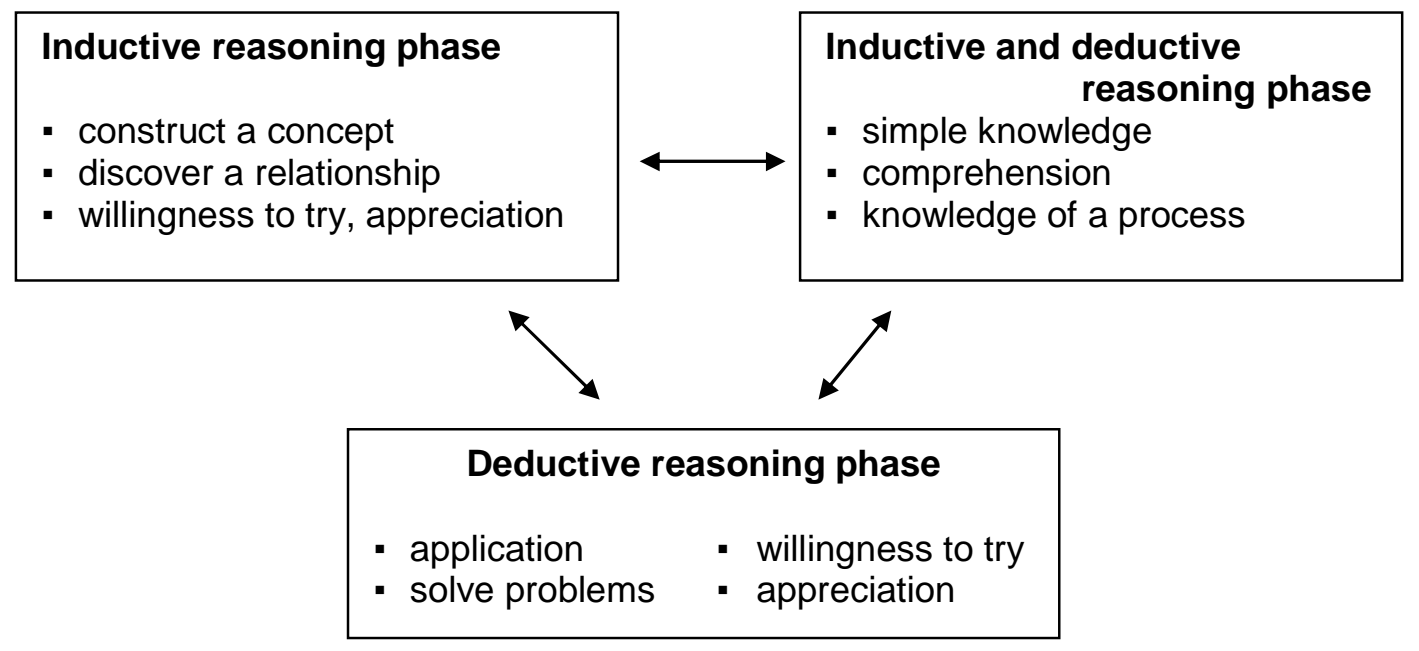

Figure 1. A guided problem-solving teaching model.

learners to construct a concept such as the concept of a quadratic equation or a cyclic quadrilateral, or discover a discoverable relationship, for example, a perfect square is always greater than or equal to zero. For the former, the use of an 'examples and non-examples approach' could be useful.

The inductive and deductive reasoning phase is the intermediate phase, which focuses on the teaching of simple knowledge and algorithms. While the basis for learning meaningful mathematics is to help learners construct concepts and to discover relationships, learners also have to remember conventional names for these concepts and relationships. They should therefore be exposed, by means of a direct instruction process, to mathematical information which they are required to remember. Requisite information includes the following examples.

- Simple knowledge: recalling specific responses (not a multi-step process) to specific stimuli. Here learning activities should target the end product, namely, the correct statement of the simple knowledge.

Example: State the standard form of the quadratic equation.

- Comprehension: extracting and interpreting meaning from symbolic representations.

Example: What do the following mean to you?

(a) $x(x+1)=12$

(b) $a . b=0 \Rightarrow a=0$ or $b=0$.

- Knowledge of a process: engaging in an algorithm or multi-step process. Here, learning activities should aim to help learners achieve algorithmic skills.
Example: Outline the procedure to solve a quadratic equation by the factorisation technique.

The direct instruction process for teaching of simple knowledge, comprehension and knowledge of a process should also have a number of appropriate stages. Guided instructional activities could be designed to stimulate a willingness to attempt the mastery of simple knowledge, and promote comprehension and algorithmic skills.

In the final phase, deductive reasoning is required. Cangelosi (1996: 157) has suggested that "...deductive reasoning is the cognitive process by which people determine whether what they know about a concept or abstract relationship is applicable to some unique situation". Therefore, in this phase, the rules of logic have to be adhered to. Application lessons require learners to put into practice previously developed or acquired concepts, relationships, information and algorithms. Learners must determine whether or not any of these may apply to a problem. Therefore, activities which stimulate learners to reason deductively should be planned in this phase.

The guided problem-solving model could be useful in addressing and rectifying the recurring issues that contribute to poor performance amongst matriculation candidates. There are two simultaneous processes in this model, one an interplay, and the other a progression or drift to deduction. Since the FET Curriculum Statements for Mathematics advocate that knowledge integrates theory, skills and values, it calls for a development towards deductive reasoning.

The guided problem-solving model is based on the theory of moving from the known to the 
unknown, taking into consideration how conceptual development occurs. A movement from the inductive reasoning phase towards the next two phases requires the gradual development of schemata and networks in the brain. Schemata provide a mental framework for understanding and remembering information (Bartlett, 1958). Schemata or mental models provide meaning and organisation to experiences, and allow the individual to go beyond the given information (Bruner, 1973). The nature of expertise is largely due to the possession of schemata that guide perception and problem-solving (Chi, Glaser \& Farr, 1988).

If this is accepted then it seems that the facilitation of the learning process in mathematics needs to focus on the development and modification of schemata. The movement in phases within the guided problem-solving model is characterised by greater abstraction. During each of the phases, well formulated problems (or questions) should form an integral part of the design of learning activities. The educator has to design suitable learning activities to focus on the relevant aspects indicated in each of the phases. These learning activities should guide learners to attain the relevant objectives or intended outcomes for each phase.

A task analysis approach, discussed below, could assist in the design of such activities. To help learners improve their understanding of mathematical concepts, activities should be designed which encourage them to explore, generalise, explain, argue and finally prove. Commenting on the importance of getting learners to explain and justify their reasoning, Jones (2000) indicates

... the requirement to explain and justify

their reasoning requires students to make

the difficult transition from a computational view to a view that conceives of mathematics as a field of intricately related structures. (2000: 58)

The design of four tasks, which aim to facilitate such a transition, will be explained in this paper.

Task analysis approach embedded within a teaching model for mathematics

The previous section led to the conclusion that a task analysis approach could be used to develop component knowledge and skills for certain problem types within the framework of the guided problem-solving model. Task analysis has two useful functions (Usability First, 2005):
- It refers to a set of methods for decomposing tasks into subtasks to understand procedures better and provide support for those tasks.

- It is useful for spotting potential errors that could arise from steps in the process which could be difficult or confusing.

The second point indicates that task analysis has the potential to serve a predictive function, such as identifying possible impediments to learning. Task analysis for the purpose of instructional design " $\ldots$ is a process of analysing and articulating the kind of learning that you expect the learners to know how to perform" (Jonassen, Tessmer \& Hannum, 1999: 3). This implies that within the framework of the FET National Curriculum Statements for Mathematics (Department of Education, 2003), task analysis is useful to design baseline and diagnostic assessments. Baseline assessment is used to establish what learners know or already can do, and therefore helps in the planning of activities. Diagnostic assessment is used to discover the cause or causes of a learning barrier, and "therefore assists in deciding on support strategies" (Department of Education, 2003: 64).

The performance of a task analysis on typical examination questions could be useful to educators when designing instruction. It could be used to:

- determine the instructional goals and objectives,

- define and describe (in detail) the tasks and sub-tasks,

- $\quad$ specify the required type of knowledge,

- select learning outcomes appropriate for instructional development,

- prioritise and sequence tasks,

- determine instructional activities and strategies that foster learning,

- select appropriate media and learning environments, and

- construct performance assessments and evaluation.

Jonassen et al. (1999) noted that the task analysis process has five distinct functions:

- classification of tasks according to learning outcomes,

- identifying or generating a list of tasks,

- prioritising tasks and choosing those that are more feasible and appropriate,

- identifying and describing the components of the tasks, and

- defining the sequence in which instruction should occur to best facilitate learning. 
However, for these functions to be realised, the assumption is that the educator is competent in the subject content.

\section{Learner-centred activities derived from the task analysis}

Previously built-up understanding could either hinder or promote the understanding of new ideas Davis, 1992); this has implications for both learning and teaching. Educators should therefore make efforts to determine whether learners have the relevant prior knowledge or abilities required for the understanding of new ideas. The task analysis and APOS approach - identifying required actions (manipulative skills), processes, objects and schema - can be of benefit to educators. There are a number of possibilities that this approach opens up for professional training, including:

- the design of spot tests or pre-tests,

- the restructuring of questions or problems to make them more learner-centred, and

- the development of summaries and strategies to aid problem-solving.

- A task analysis approach could also help to pinpoint the different competencies required by mathematics educators to do these. The following sections note required competencies, and illustrate some of the possible situations in which task analysis could be useful.

\section{Spot tests or pre-tests}

Mathematics educators should have some way of determining whether or not the requisite prior knowledge, skills and abilities are really in place. This could be done by oral questioning or designing a spot test for learners to complete. Spot tests could also be used to determine whether learners are able to outline the key steps in the proof of a theorem, based on the context of a given diagram. A time limit should be placed on the completion of such tests, and each test should be followed by a class discussion. Spot tests or pretests could thus be useful for baseline and diagnostic assessments.

\section{Task 1}

Suppose that the objective or specific outcome for the first lesson unit on solution of quadratic equations is:

At the end of the lesson learners should be able to solve quadratic equations of the type $3 x^{2}+2 x=5$ by using the factorisation technique.

Required for this lesson unit:

1.1 Pinpoint the prior knowledge / abilities assumed.

1.2 For the prior knowledge / abilities indicated in (1.1), design a spot test that could help you to determine whether learners have these knowledge and abilities.

A possible response to Task 1:

1.1 Prior knowledge/abilities assumed:

a) Ability to factorise quadratic polynomials of the types $x^{2}-4$; $x^{2}+3 x ; 3 x^{2}+2 x-5$

b) Knowledge and application of the zero product rule:

For real numbers $p$ and $q$, $p . q=0 \Leftrightarrow p=0$ or $q=0$.

c) Ability to solve linear equations of the type: $2 x+5=0 ; 3 x-2=0$.

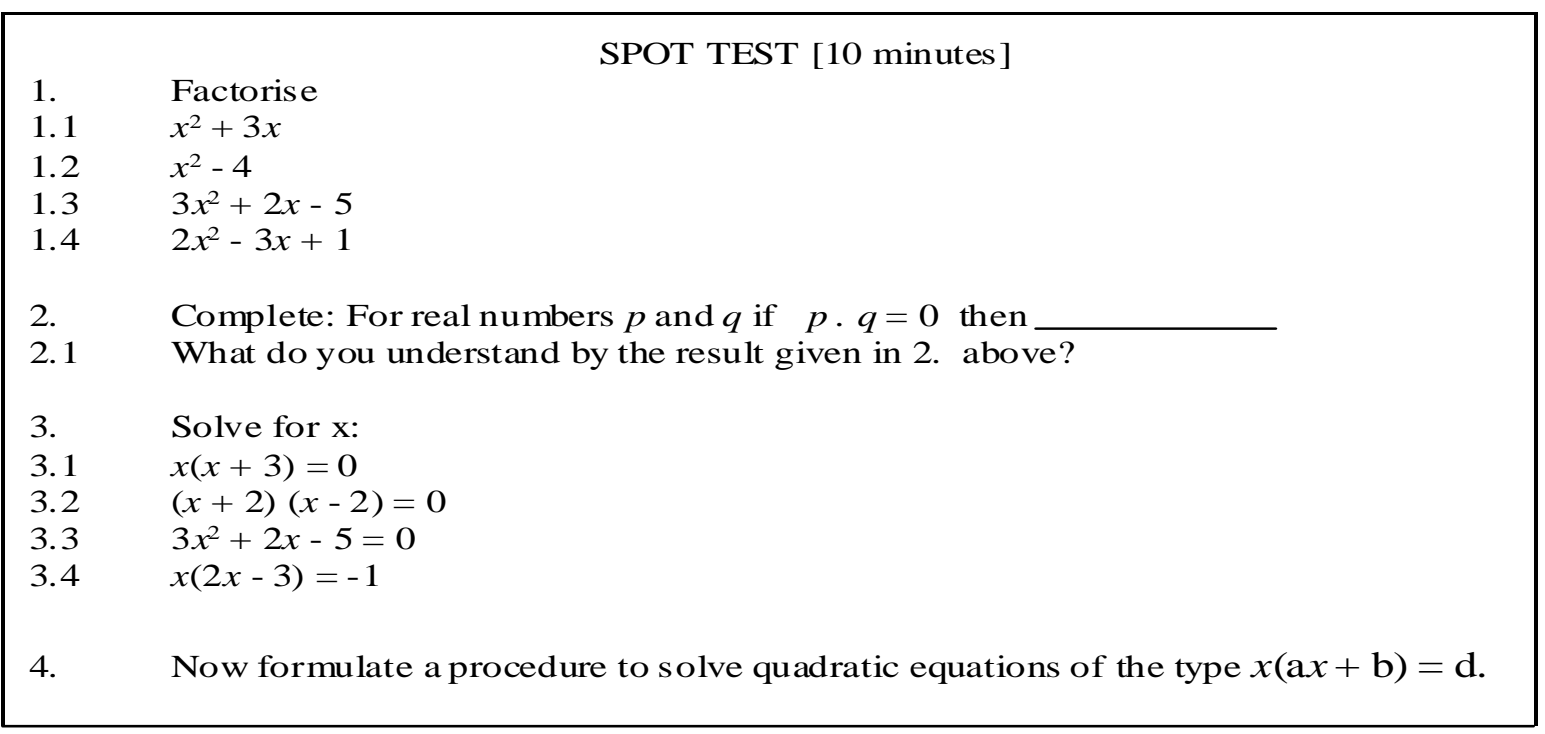

Box 2. Example of a spot test. 
An example of a spot test that may be used to pinpoint prior knowledge and abilities (1.1) is illustrated in Box 2.

In the context of the guided problem-solving model, this spot test targets the inductive and deductive reasoning phase. Note that question 1.4 of this test is designed to formulate an algorithm (which links component parts) to solve quadratic equations. A class discussion could focus on connecting, refining and justifying steps in the algorithm.

\section{Restructuring a problem - an example from analytical geometry}

Educators should be able to work out problems based on the different sections of the syllabus. For a given problem they should be able to pinpoint the relevant prior knowledge and abilities required by learners. If the problem is deemed to be difficult for learners, educators could use this information to restructure the problem, with the aim of helping them solve it. This restructuring could include:

(a) rewriting the given information clearly,

(b) providing a diagram or figure to support the visualisation of the given information, and

(c) formulating suitable sub-questions to enable learners solve the problem.

A typical example of what would need to be done is illustrated in Task 2 .

\section{Task 2}

Study the problem:

Determine the equation of the tangent to the circle $x^{2}-2 x+y^{2}+4 y=5$ at the point $(-2 ;-1)$.

Now answer the following questions based on the above problem.

2.1 Solve the above problem.

2.2 Write down the relevant prior knowledge/abilities required by learners to work out the problem.

2.3 Restructure the above problem with the aim of helping learners to solve the problem.

An example of a possible restructuring of the given problem is provided in Box 3. The original problem required learners to integrate different component tasks into a solution. If the relevant component knowledge and abilities are in place, learners should also be exposed to a subsequent task such as the following:

Consider the problem: The straight line $y+2 x-10=0$ intersects with the circle with centre $O$ (the origin) at points $A$ and $T$, where $A$ is on the $x$-axis. Determine the co-ordinates of $D$ if $O D$ is perpendicular to $A T$ and D lies on AT. Write down the key steps that lead to a solution of this problem.

In the context of the guided problem-solving teaching model such activities help to make the transition to the deductive reasoning phase.

\section{Deductive reasoning - an example from geometry} Examiners' reports indicated that learners perform poorly in the proofs of geometry theorems. This could be as a result of the way in which these theorems were introduced to learners. For example, a theorem was stated and then the proof was given. Learners also have many misconceptions and make common errors that relate to the application of the theorem's statement.

A mathematics educator could help learners by

(a) pinpointing the relevant prior knowledge/ abilities required by learners to prove the theorem [first analyse the proof of the theorem], (b) designing a worksheet that helps learners arrive

1. The figure shows the circle defined
by the equation $x^{2}-2 x+y^{2}+4 y=5$.
The line $l$ is a tangent to the circle at
the point $\mathrm{M}(-2 ;-1)$.
Determine the co-ordinates of $\mathrm{P}$, the centre of
the circle.
1.2 Calculate the gradient of $\mathrm{MP}$.
1.3 Why is MP perpendicular to the line $l$ ?
1.4 Now determine the defining equation of the
tangent $l$.

Box 3. Restructuring a problem - an example. 
deductively at the statement [draw the relevant diagram, write up the given information and formulate suitable lead-on questions by using the relevant prior knowledge/abilities],

(c) formalising the statement of the theorem [write the statement in the "if.....then" form and use diagrams to illustrate the "cause and effect"], and

(d) giving quick and simple applications based on the pre-requisites to apply the statement of the theorem.

Task 3 was designed to help mathematics educators develop these competencies.

\section{Task 3}

The competencies stated above could be developed through the following example:

3.1 Complete the following statement of a theorem: If two triangles are equiangular then their...

3.2 For the statement of the theorem in (3.1) above you are required to do the following:

a) List the prior knowledge/abilities required by learners to prove the statement.

b) Design a worksheet which could enable learners to deductively arrive at the statement.

1. $\quad$ In $\triangle \mathrm{ABC}$ and $\triangle \mathrm{PQR}$

$$
\begin{aligned}
\hat{\mathrm{A}} & =\hat{\mathrm{P}} \\
\hat{\mathrm{B}} & =\hat{\mathrm{Q}} \\
\text { and } \hat{\mathrm{C}} & =\hat{\mathrm{R}}
\end{aligned}
$$
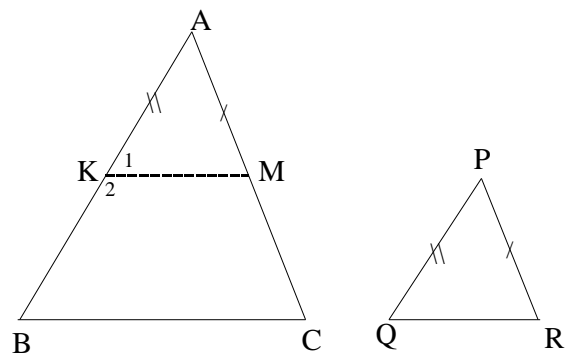

1.1 On $A B$ point $K$ is marked so that $A K=P Q$.

On $A C$ point $M$ is marked so that $A M=P R$. $K M$ is joined.

1.2 Prove that $\triangle \mathrm{AKM} \equiv \Delta \mathrm{PQR}$.

1.3 Why is $\hat{\mathrm{K}}_{1}=\hat{\mathrm{Q}}$ ?

1.4 Now show that $\hat{\mathrm{K}}_{1}=\hat{\mathrm{B}}$.

1.5 Deduce that $\mathrm{KM} \| \mathrm{BC}$.

1.6 Why is $\frac{\mathrm{AK}}{\mathrm{AB}}=\frac{\mathrm{AM}}{\mathrm{AC}}$ ?

1.7 Now show that $\frac{\mathrm{PQ}}{\mathrm{AB}}=\frac{\mathrm{PR}}{\mathrm{AC}}$.

1.8 Point $E$ is marked on $B A$ such that $B E=Q P$ and point $F$ is marked on $B C$ such that $B F=Q R$. Write down equal ratios similar to those in 1.7 by using $\mathrm{QP}, \mathrm{BA}, \mathrm{QR}$ and $\mathrm{BC}$.

1.9 Use the ratios from 1.7 and 1.8 to write down ratios that relate all the sides of $\triangle \mathrm{PQR}$ and $\triangle \mathrm{ABC}$.

1.10 Now write down a general statement about the corresponding sides of equiangular triangles.

Box 4. Examble of a worksheet - deductive response (Task 3.2b). 


\section{Aneshkumar Maharaj}

c) Briefly outline strategies to eliminate common errors / misconceptions relating to the application of this theorem.

An example of a response to (b), after the prior knowledge and abilities were detected, is provided in an example worksheet (Box 4).

To get learners to integrate component tasks into a solution, such an activity should be followed by a suitable spot test, for example see Box 5 .

These activities are designed to make the transition towards the deductive reasoning phase of the guided problem-solving teaching model.

\section{Develop summaries and strategies to support problem-solving}

Summaries and strategies help to expand and improve the connections of the network in the brain. The devising of plans should be taught, as it is a form of representation, and an important part of problem-solving. To develop into successful problem-solvers learners need to spend time analysing problems and the directions that could be taken (Fernandez, Hadaway \& Wilson, 1994). This should lead to the development of frameworks (schemata) aimed at pinpointing the different phases and the processes that are necessary when solving particular types of problems. Such schemata illustrate that the development of mathematical thinking is a sequence of ever more advanced transitions from operational to structural outlooks. A mathematics educator should facilitate the development of summaries and teachinglearning strategies in mathematics to help learners. These strategies could include frameworks or schemata, which involves representing, connecting and justifying procedures. Task 4 has been designed as an example of what could be done to help educators develop summaries and strategies.
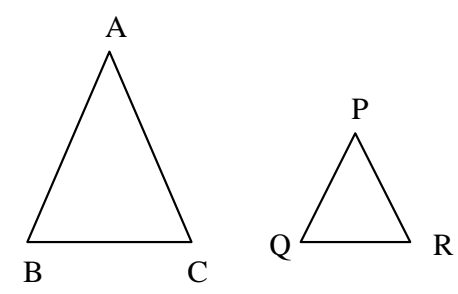

In triangles $\mathrm{ABC}$ and $\mathrm{PQR}$ $\hat{\mathrm{A}}=\hat{\mathrm{P}}, \hat{\mathrm{B}}=\hat{\mathrm{Q}}$ and $\hat{\mathrm{C}}=\hat{\mathrm{R}}$.

Write down the key steps to prove the theorem that states $\frac{\mathrm{AB}}{\mathrm{PQ}}=\frac{\mathrm{BC}}{\mathrm{QR}}$.

Box 5. Example of a spot test.

\section{Task 4}

For any one of the sections in the mathematics syllabus for grades 10 to 12 , give an example of a summary or strategy that you helped your learners to develop. The following should be indicated.

4.1 The grade and topic/section.

4.2 Full details of the summary or strategy.

4.3 Has this summary or strategy helped your learners improve their performance in mathematics? Motivate your response.

An example of a strategy that may be used to solve equations is illustrated in Figure 2. The schema for solving equations algebraically associates particular problem-solving procedures with each of the algebraic objects (linear, quadratic and cubic equations). Such a schema requires that learners first identify the type of equation (structure) they are confronted with. This should be followed by an algorithm which outlines a particular procedure, for example, the factorisation technique for quadratic equations. Such a schema can help learners to "... understand, deal with,

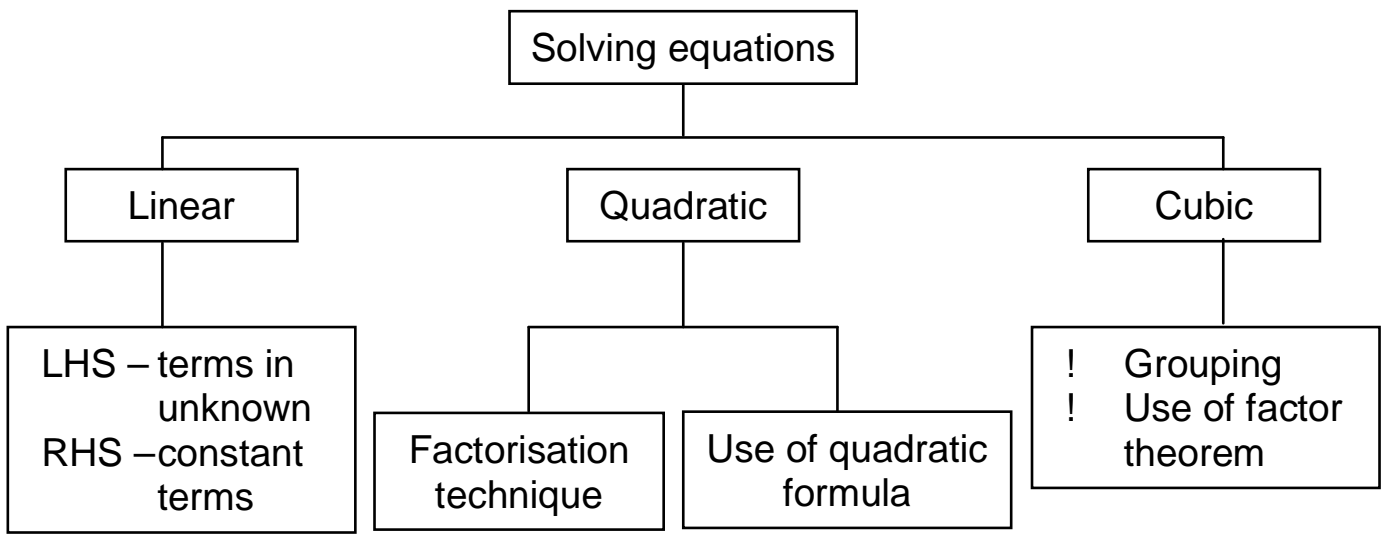

Figure 2. Schema for solving equations algebraically. 
organise, or make sense out of a perceived problem situation" (Dubinsky 1991: 102), as it includes a more or less coherent collection of objects and processes. In this case, the task entails solving equations - of degree one, two or three - in one unknown. By focussing on the structural nature of such schemata, explicit descriptions of possible relationships between schemata can be detected. Instruction should therefore focus on the need for, the usefulness of and the development of such schemata to aid thinking and bringing into play encoded labels for algorithms.

\section{Conclusion}

The task analysis approach, with a focus on detecting required actions, processes, objects and schema, could be used to design process-based learner-centred activities within the framework of the guided problem-solving model. It is evident from the four tasks outlined in this paper that this approach requires competent educators who have a sound knowledge of the content to be taught. The approach can be used to design questions and activities that address the typical common errors, misconceptions and difficulties of learners as identified in the examiners' reports. Some examination questions contain complex component tasks. Therefore, there is also a need to follow-up with activities that require learners to integrate component tasks. Within the framework of the guided problem-solving model, such activities target the deductive reasoning phase.

\section{References}

Bartlett, F.C. (1958). Thinking. New York: Basic Books.

Bruner, J. (1973). Going Beyond the Information Given. New York: Norton.

Cangelosi, J.S. (1996). Teaching Mathematics in Secondary and Middle School: An Interactive Approach. Second edition. New Jersey: Prentice Hill.

Chi, M., Glaser, R. \& Farr, M. (1988). The Nature of Expertise. Hillsdale, NJ: Erlbaum.

Davis, R.B. (1992). Understanding "Understanding". Journal of Mathematical Behavior, $11,225-241$.

Department of Education (2003). Mathematics National Curriculum Statement Grades 10-12 (General). Pretoria: Government Printer.

Dubinsky, E. (1991). Reflective abstraction in advanced mathematical thinking. In D. Tall
(Ed.), Advanced mathematical thinking (pp 95123). London: Kluwer Academic Publishers.

Dubinsky, E., Weller, K., McDonald M.A. \& Brown A. (2005). Some Historical Issues and Paradoxes regarding the Concept of Infinity: An APOS Analysis: Part 2. Educational Studies in Mathematics, 60, 253-266.

Fernandez, M.L., Hadaway, N., \& Wilson, J.W. (1994). Problem-solving: Managing it all. Mathematics Teacher, 87(3), 195-199.

Gauteng Department of Education (2000). Matriculation Examiners' Report for Mathematics 1999.

Gauteng Department of Education (2001). Matriculation Examiners' Report for Mathematics 2000.

Gauteng Department of Education (2002). Matriculation Examiners' Report for Mathematics 2001.

Gauteng Department of Education (2003). Matriculation Examiners' Report for Mathematics 2002.

Hiebert, J. \& Carpenter, T. P. (1992). Learning and Teaching with Understanding. In D.A. Grouws (Ed.), Handbook of Research on Mathematics Teaching and Learning (pp 65-97). New York: Macmillan Publishing Company.

Jonassen, D.H., Tessmer, M., Hannum, W.H. (1999). Task Analysis Methods for Instructional Design. Mahwah, New Jersey: Lawrence Erlbaum Associates.

Jones, K. (2000). The student experience of mathematical proof at university level. International Journal of Mathematical Education in Science and Technology, 31(1), 53-60.

KwaZulu-Natal Department of Education (2000). Matriculation Examiners' Report for Mathematics 1999.

KwaZulu-Natal Department of Education (2001). Matriculation Examiners' Report for Mathematics 2000.

KwaZulu-Natal Department of Education (2002). Matriculation Examiners' Report for Mathematics 2001.

Mason, J. (2000). Asking mathematical questions mathematically. International Journal of Mathematical Education in Science and Technology, 31(1), 97-111.

Usability First (2005). Usability Glossary: task analysis. Retrieved March 9, 2005, from http://www.usabilityfirst.com/glossary/main.cgi ?function=display_term\&term_id=294 